\title{
Typing and evaluating heat resistance of Bacillus cereus sensu stricto isolated from the processing environment of powdered infant formula
}

\author{
Kejin Zhuang, ${ }^{1}$ Hongfu Li, ${ }^{1}$ Ziwei Zhang, ${ }^{1}$ Shuang Wu, ${ }^{1}$ Yashuo Zhang, ${ }^{1}$ Edward M. Fox, ${ }^{2,3}$ Chaoxin Man, ${ }^{1 *}$ \\ and Yujun Jiang ${ }^{1 *}$ \\ ${ }^{1}$ Key Laboratory of Dairy Science, Ministry of Education, Department of Food Science, Northeast Agricultural University, Harbin, 150030, China \\ ${ }^{2}$ Department of Applied Sciences, Northumbria University, Newcastle upon Tyne, United Kingdom NE 98 \\ ${ }^{3}$ CSIRO Agriculture and Food, Werribee, VIC 3030, Australia
}

\section{ABSTRACT}

Bacillus cereus sensu lato is one of the most harmful bacterial groups affecting the quality and safety of powdered infant formula (PIF). In this study, samples were collected from the raw materials and processing environments of PIF. A total of 84 isolates were identified as Bacillus cereus sensu stricto (B. cereus s. s.) by 16S rRNA analysis, molecular typing technology, and physiological and biochemical tests. The $84 \mathrm{~B}$. cereus s. s. strains were assigned to panC group II, group III, and group IV. Then, the 7 housekeeping genes $g l p F$, gmk, ilvD, pta, pur, pycA, and tpi were selected for multilocus sequence typing. Results showed that the 84 isolates were clustered into 24 sequence types (ST), and 14 novel ST were detected. Among the $24 \mathrm{ST}$, ST999 $(19 / 84,22.62 \%)$ and ST1343 $(13 / 84,15.48 \%)$ predominated. The correlation between processing areas and ST showed that the processing environments of the production and packing areas were the most susceptible to contamination by $B$. cereus $s$. $s$. Spores of these ST showed different heat resistance phenotypes evaluated by the analysis of $\mathrm{D}_{\mathrm{T}}$ (time in minutes of spore decimal reduction at each temperature) and $\mathrm{Z}$ values (temperature increase required to reduce the $D_{T}$ value to one-tenth of the original). Spores from group III according to panC gene analysis were the most heat resistant. These findings will help us to better understand B. cereus s. s. contamination and control in PIF processing environments.

Key words: powdered infant formula, Bacillus cereus sensu stricto, processing environments, heat resistance

Received January 29, 2019.

Accepted May 13, 2019.

*Corresponding authors: mcxwh2006@qq.com and yujun_jiang@ 163.com

\section{INTRODUCTION}

Powdered infant formula (PIF) contains the nutrients necessary for the growth and development of infants and it is an excellent substitute for human milk (Kent et al., 2015). In recent years, the quality and safety of PIF are increasing concerns because they are closely related to infant health. Several researchers have reported that pathogenic microorganisms are present in PIF (Fei et al., 2015; Liu et al., 2018).

Processing PIF involves high-temperature sterilization and spray drying, with the vegetative cells of pathogenic bacteria being killed during processing. However, because Bacillus cereus shows strong tolerance or resistance to heat, dryness, and disinfectants, heat-resistant spores produced by B. cereus group species may not be eliminated completely (Kumari and Sarkar, 2016). These spores may germinate into vegetative cells and form biofilms that produce toxins or spoilage enzymes (Scheldeman et al., 2006; Esteban et al., 2013). Additionally, biofilms may lead to prolonged presence of $B$. cereus group strains in the processing environment, and produce heat-stable enzymes, which may have negative implications for product quality (Teh et al., 2012). Therefore, the presence of sporeformers with strong heat resistance could be an important factor in deterioration of PIF quality, leading to defects in flavor and function and causing spoilage of PIF or food poisoning (Hayrapetyan et al., 2016). These properties allow B. cereus to be introduced into PIF from raw materials and processing environments, which cause foodborne illness and have negative effects on human health (Mckillip et al., 2014; Patiño-Navarrete and Sanchis, 2017). Thus, the contamination of B. cereus in PIF should be controlled strictly.

The B. cereus group, also known as B. cereus sensu lato, is composed of gram-positive, rod-shaped, sporeforming aerobic bacteria that exist widely in the environment (Luu-Thi et al., 2014). This group comprises 11 species (Liu et al., 2017), B. cereus sensu stricto 
(B. cereus s. s.), B. anthracis, B. thuringiensis, B. mycoides, B. pseudomycoides, B. weihenstephanensis (Lechner et al., 1998), B. cytotoxicus, B. toyonensis, B. gaemokensis, B. manliponensis, and B. bingmayongensis (Giffel and Beumer, 1999). The identification and typing of the $B$. cereus group can provide useful insights into the contamination routes of PIF. However, species of the $B$. cereus group cannot be confirmed based on traditional detection methods and phenotyping technologies, because the physiological-biological properties and $16 \mathrm{~S}$ rRNA sequences of species of the $B$. cereus group are similar (Liu et al., 2018). Therefore, molecular typing methods, such as panC gene analysis (Guinebretière et al., 2013) and multilocus sequence typing (MLST; Maiden et al., 2013) should be combined with traditional methods to differentiate diverse species of the B. cereus group (Jung et al., 2011; Otlewska et al., 2013; Castiaux et al., 2014).

The pan $C$ gene is a housekeeping gene of the $B$. cereus group that encodes pantothenate- $\beta$-alanine ligase. This gene is associated with the growth temperature and heat resistance of the $B$. cereus group. The pan $C$ gene has been used to distinguish different species of the $B$. cereus group with similar genetic and physiologicalbiochemical characteristics (Guinebretière et al., 2008). According to panC genotyping, different isolates in the $B$. cereus group can be divided into 7 groups (I to VII) with different phenotypic characteristics (Guinebretière et al., 2008). Candelon et al. (2004) identified 12 isolates from the $B$. cereus group by $g d p D, \operatorname{pan} C$, and $p l c R$ gene typing and demonstrated that the pan $C$ gene was more effective than $g d p D$ or $p l c R$ to classify $B$. cereus strains.

Multilocus sequence typing is one of the most popular typing methods for bacterial species (Santos et al., 2012), and it is a powerful method for tracing analysis as well (Yang et al., 2017). Genetic evolutions can be determined by comparative analysis of alleles (Forsythe et al., 2014; Hammerum et al., 2015); MLST facilitates gene analysis and the study of genetic evolution of bacteria by amplifying housekeeping genes (Drewnowska and Swiecicka, 2013; Lin et al., 2017). Unique sequences of each housekeeping gene are assigned an allele number (allele), the combination of which relates to a specific sequence type $(\mathbf{S T})$. These alleles and ST genotypes are curated and the alleles can be compared online (Pérez-Losada et al., 2013).

In this study, we combined panC gene typing with MLST analysis to characterize $B$. cereus s. s. isolated from the raw materials and processing environments of PIF facilities. The heat resistance of $B$. cereus s. s. in different ST and phylogenetic groups was explored to enhance our understanding of heat resistance in $B$. cereus s. s. subgroups, which can inform strategies to reduce the contamination of PIF.

\section{MATERIALS AND METHODS}

\section{Culture Media and Chemical Reagents}

Phosphate-buffered saline, mannitol-egg yolkpolymyxin (MYP) agar, tryptose soy agar (TSA), Luria-Bertani (LB) broth medium, nutrient agar (NA) medium, casein agar, alkaline bromide, peptone, beef extract powder, and agar were purchased from Hope Bio-Technology (Qingdao, China). Sheep blood was obtained from Elite Media Co., Ltd. (Shanghai, China). Methanol, $\mathrm{NaCl}, \mathrm{MnSO}_{4} \cdot \mathrm{H}_{2} \mathrm{O}, \mathrm{MgSO}_{4} \cdot 7 \mathrm{H}_{2} \mathrm{O}$, $\mathrm{FeSO}_{4} \cdot 7 \mathrm{H}_{2} \mathrm{O}, \mathrm{KCl}$, and $\mathrm{CaCl}_{2} \cdot 2 \mathrm{H}_{2} \mathrm{O}$ were of analytical grade and purchased from Aladdin Reagent Co. Ltd. (Shanghai, China).

\section{Sample Collection}

Sample collection was conducted in a typical wetprocessing PIF factory located in northeast China. Samples were collected once a month from November 2014 to June 2015. Based on the PIF processing workflow, 6 sampling locations were selected for sample collection: (A) raw materials area, (B) pretreatment area, (C) production area, (D) big bag filling area, (E) can filling area, and (F) packaging area. Samples of raw materials were collected from 14 categories. Samples collected from the processing environments (B to $\mathrm{F}$ ) included air samples and samples from the surface of production equipment, the floor, and the hands, shoes, and clothes of operators. The details of raw materials and numbers of samples collected from each sampling locations are summarized in Table 1.

Around $200 \mathrm{~g}$ of each type of raw material was collected and stored in low-temperature sampling boxes. Samples were returned to the laboratory for analysis as quickly as possible. All raw material samples were analyzed in triplicate. A microbe air sampler fitted with an aseptic Millipore filter (JWL-SWI, Sennon Technologies, Beijing, China) was used to collect bacteria in the air of processing environments (B to $\mathrm{F}$ ). The number of air samples was determined according to the sampling area, and air samples were taken per $10 \mathrm{~m}^{2}$. The sampler was placed on the tripod about $1 \mathrm{~m}$ above each sampling point (the center and 4 corners to the east, west, north, and south of sampling area), the sampling time was about $2.5 \mathrm{~min}$, and the inlet flow velocity was $200 \mathrm{~L} / \mathrm{min}$. Three samples were taken at each sampling point for determination of bacteria. A total of 15 air samples were collected from processing environments B 
Table 1. Basic information of samples collection

\begin{tabular}{llll}
\hline $\begin{array}{l}\text { Sampling } \\
\text { point }\end{array}$ & Sampling area & Type of samples & $\begin{array}{c}\text { No. of } \\
\text { samples }\end{array}$ \\
\hline A & Raw materials & $\begin{array}{l}\text { Raw milk, } \alpha \text {-lactalbumin, whey protein concentrate, casein phosphopeptide, calcium } \\
\text { carbonate, nutrients, lactose, potassium chloride, vitamins, nucleotides, minerals, fructo- }\end{array}$ & 42 \\
oligosaccharide, lactoferrin, palm oil & Air samples and surface of production equipment, floor, and hand, shoes and clothes of \\
B & Pre-treatment & operators & 48 \\
C & Production & & 54 \\
D & Big bag filling & & 60 \\
E & Can filling & & \\
F & Packaging & & \\
\hline
\end{tabular}

to F. After sampling, the filters that absorbed bacteria in the samplers were transferred into sterile chambers and stored at $4^{\circ} \mathrm{C}$.

Sterile cotton swabs moistened with sterile PBS were used to collect bacteria on the production equipment, floor, and hand, shoes, and clothes of operators. The areas most likely to be contaminated on the surface of equipment and the floor were chosen as sampling points. The area of each sampling point was $10 \times 10$ $\mathrm{cm}^{2}$. The sampling area of hands, shoes, and clothes of each operator was $5 \times 10 \mathrm{~cm}^{2}$. After sampling, the swabs were placed into sterile containers and stored at $4^{\circ} \mathrm{C}$.

\section{Bacterial Isolation}

Samples from raw materials and the surfaces of production equipment were serial diluted several times with PBS and spread onto MYP agar. Then, the plates were inverted and bacteria were incubated at $30^{\circ} \mathrm{C}$ for $24 \mathrm{~h}$. In general, TSA and LB broth medium are universal media for bacterial culture. Therefore, isolates from air samples were collected on TSA. Afterward, colonies were cultured in LB broth medium for $12 \mathrm{~h}$, followed by plating and incubation on MYP agar. Rough or dry colonies surrounded by egg yolk precipitation on a pink MYP agar background were considered to be presumptive positive $B$. cereus group isolates.

\section{DNA Extraction}

We extracted DNA from overnight cultures grown in LB liquid medium using a TIANamp Bacteria DNA Extraction Kit (Tiangen Biotech, Beijing, China) according to the manufacturer's instructions for grampositive bacteria.

\section{Identification of Isolates}

16S rRNA Analysis. The DNA was amplified using 16S rRNA sequencing with the universal prim- ers 515F (5'-GTGCCAGCMGCCGCGG-3') and 907R (5'-CCGTCAATTCMTTTRAGTTT-3') (Wu et al., 2018). The PCR products were purified using a PCR clean-up kit (TaKaRa, Dalian, China). Then, the purified PCR products were directly sequenced by SinoGenoMax Co. Ltd. (Beijing, China). The 16S rRNA sequences were aligned by the BLASTN algorithm in the GenBank database (http://www.ncbi.nlm.nih.gov/ BLAST/).

panC Gene Sequence Analysis. The PCR amplification of the pan $C$ gene was performed using the primers TYGGTTTTGTYCCAACRATGG and CATAATCTACAGTGCCTTTCG, which were provided by an online typing tool (https://www.tools.symprevius .org/bcereus/english.php). The PCR reaction mixture and conditions were described by Guinebretière et al. (2013). The PCR products were sequenced by SinoGenoMax Co. Ltd.

Hemolysis and Caseinase Tests. To further confirm these isolates, a single colony on NA medium was picked for physiological and biochemical tests (hemolysis, caseinase, and parasporal crystal tests). Because $B$. cereus s. $s$. has $\beta$-hemolytic activity and the ability to hydrolyze casein, whereas $B$. anthracis does not have these abilities, the hemolysis and caseinase tests can be used to distinguish between $B$. cereus s. s. and $B$. anthracis. The enzymatic activity of hemolysis and caseinase were screened using selective medium, TSA with $5 \%$ sheep blood and casein agar, and incubated at $37^{\circ} \mathrm{C}$ for $24 \mathrm{~h}$.

Parasporal Crystal Test. Compared with B. cereus s. s., B. thuringiensis can produce parasporal crystals. Thus, B. cereus s. s. and B. thuringiensis can be distinguished by parasporal crystal test. The bacterial culture was spread onto NA medium supplemented with $\mathrm{MnSO}_{4} \cdot \mathrm{H}_{2} \mathrm{O}(0.0308 \mathrm{~g} / \mathrm{L})$ and incubated at $30^{\circ} \mathrm{C}$ for $24 \mathrm{~h}$. The plates were stored at room temperature for $3 \mathrm{~d}$. The culture was picked up on a glass slide with distilled water. After drying and fixing the slide, methanol was added for $30 \mathrm{~s}$. The bacteria were stained with $0.5 \%$ alkaline bromide and put on the flame for 
$1 \mathrm{~min}$. The alkaline bromide was washed off and the bacteria were stained again. The slides were examined for the presence of parasporal crystals using an optical microscope (BA200, Motic China Group Co., Ltd., Xiamen, China).

\section{MLST Analysis}

Seven housekeeping genes, $g l p F$ (glycerol uptake facilitator protein), gmk (putative guanylate kinase), $i l v D$ (dihydroxy-acid dehydratase), pta (phosphate acetyltransferase), pur (phosphoribosylamino-imidazolecarboxamide), $p y c A$ (pyruvate carboxylase), and tpi (triosephosphate isomerase), were chosen according to the B. cereus MLST database (http://pubmlst.org/ bcereus/). These 7 housekeeping genes are used to classify the $B$. cereus group because they are single copy, have high discriminatory power, and are not subject to lateral transfer or genetic recombination (Priest et al., 2004; Zahner et al., 2013; Liu et al., 2017). The PCR products were sequenced by SinoGenoMax Co. Ltd. The sequences of the 7 housekeeping genes were assigned to specific allele numbers by referencing the $B$. cereus MLST database, and ST were numbered based on the combination of 7 housekeeping genes allele numbers. If there were no exact allele numbers or exact matches for submitted sequence or ST, a new allele number or ST was assigned.

The nucleotide diversity indices of 7 loci, including the number of alleles, the number of polymorphic sites, nucleotide diversity per site $(\pi)$, the mean $\mathrm{G}+\mathrm{C}$ content, the $\mathrm{Ka} / \mathrm{Ks}$ ratio (where Ka represents the number of nonsynonymous substitutions per nonsynonymous site, and Ks represents the number of synonymous substitutions per synonymous), and Tajima's D (the deviation from the standard neutral model of evolution) were calculated with DnaSP version 5.0 software (Fei et al., 2015).

\section{Phylogenetic Analysis}

The phylogenetic relationship of the concatenated sequences $(2,829 \mathrm{bp})$ of the 7 housekeeping genes was analyzed using the maximum-likelihood algorithm in MEGA (Molecular Evolutionary Genetic Analysis) 7.0 software, with 1,000 bootstrap replicates test. To determine the interspecific phylogenetic relationship, 11 reference stains of $B$. cereus group were included (B. cereus ATCC $14579^{\mathrm{T}}$, B. anthracis ATCC $14578^{\mathrm{T}}$, B. thuringiensis ATCC $10792^{\mathrm{T}}$, B. mycoides ATCC $6462^{\mathrm{T}}$, B. pseudomycoides DSM $12442^{\mathrm{T}}$, B. weihenstephanensis DSM $11821^{\mathrm{T}}$, B. cytotoxicus NVH 391-98 ${ }^{\mathrm{T}}$, B. toyonensis BCT-7112 ${ }^{\mathrm{T}}$, B. gaemokensis $\mathrm{BL} 3-6^{\mathrm{T}}, B$. manliponensis BL $4-6^{\mathrm{T}}$, and B. bingmayongensis FJAT$\left.13831^{\mathrm{T}}\right)$.

\section{Population Genetics Analysis}

Genetic relationship and population structure among isolates were analyzed by the eBURST online tool (http: //pubmlst.org/bcereus/). Single locus variant (SLV) and double locus variants (DLV) were determined by the eBURST online tool to analyze the relationship of B. cereus s. s. ST. All ST could be divided into different clusters or singletons. The clusters of identical alleles from 6 of the 7 loci were identified as a clonal complex (CC). The ST with the highest SLV value among a CC was considered the central ST of that CC (Liu et al., 2017).

\section{Tracing Analysis}

The correlations between different ST and sample sources were analyzed by using SPSS 22.0 software (SPSS Inc./IBM Corp., Chicago, IL) to trace the isolates from the raw materials and processing environments of PIF.

\section{Heat Resistance of Different ST of B. cereus}

All isolates of each ST were evaluated for heat resistance characteristics. One hundred microliters of each culture in LB was spread on sporulation agar and incubated for 3 to $7 \mathrm{~d}$ at $37^{\circ} \mathrm{C}$ to prepare a spore suspension. The final $\mathrm{pH}$ of the sporulation agar was $7.3 \pm 0.1$ and included the following ingredients $\left(\mathrm{L}^{-1}\right)$ : peptone $(3.3$ g), beef extract powder $(1 \mathrm{~g}), \mathrm{NaCl}(1.7 \mathrm{~g}), \mathrm{MnSO}_{4} \cdot \mathrm{H}_{2} \mathrm{O}$ $(0.003 \mathrm{~g}), \mathrm{MgSO}_{4} \cdot 7 \mathrm{H}_{2} \mathrm{O}(0.51 \mathrm{~g}), \mathrm{FeSO}_{4} \cdot 7 \mathrm{H}_{2} \mathrm{O}(0.1 \mathrm{~g})$, $\mathrm{KCl}(0.97 \mathrm{~g}), \mathrm{CaCl}_{2} \cdot 2 \mathrm{H}_{2} \mathrm{O}(0.2 \mathrm{~g})$, and agar $(15 \mathrm{~g})$. The plates were observed regularly by microscope and harvested when more than $90 \%$ of the cells sporulated. The spores were collected with cold deionized water and the entire growth surface was scraped from the plate using sterile hockey spreaders and transferred to a $15-\mathrm{mL}$ centrifuge tube for centrifugation at $4,000 \times g$ for 10 min at $4^{\circ} \mathrm{C}$ (Multifuge X3R, Thermo Fisher Scientific, Waltham, MA). The supernatant was washed 3 times. Finally, the spores were resuspended in deionized water to a concentration of approximately $10^{8}$ spores $/ \mathrm{mL}$ and stored at $4^{\circ} \mathrm{C}$ for 30 to $40 \mathrm{~d}$ to obtain mature spores.

To enumerate spores, the spore suspension was heated at $80^{\circ} \mathrm{C}$ for $10 \mathrm{~min}$ to kill the remaining vegetative cells. One hundred milliliters of the spore suspension was then heated in an oil bath at 115,118 , or $121^{\circ} \mathrm{C}$ for $5,10,15$, or $20 \mathrm{~min}$. After heating, the spore suspension was quickly cooled and the number of surviving spores 
was determined by plate count on TSA (Luu-Thi et al., 2014). We calculated the time (min) of spore decimal reduction at each temperature $\left(\mathbf{D}_{\mathrm{T}}\right.$; Ziane et al., 2014$)$ using the following formula (Mafart et al., 2002):

$$
\left(\frac{t}{\mathrm{D}_{\mathrm{T}}}\right)^{K}=\log N_{0}-\log N,
$$

where $N_{0}$ is the number of spores before heat, $N$ is the number of surviving spores after heating for $t$ minutes, and $K$ is the slope of the curve of heating time (abscissa) and number of surviving spores (ordinate). The $\mathrm{Z}$ value represents the temperature increase required to reduce the $\mathrm{D}_{\mathrm{T}}$ value to one-tenth of the original. Therefore, the $\mathrm{Z}$ value was obtained from the negative reciprocal of the slope of the regression line by plotting $\log \mathrm{D}_{\mathrm{T}}$ versus temperature. All heat treatments were carried out in triplicate using the same spore suspension.

\section{Statistical Analysis}

The data were expressed as mean \pm standard deviation. The significance of differences was determined by one-way ANOVA of SPSS 22.0 software (SPSS Inc./ IBM Corp.).

\section{RESULTS}

\section{Isolation and Identification of B. cereus sensu lato}

Based on the highest similarity with known sequences of B. cereus group strains presented in GenBank of the National Center for Biotechnology Information, 84 isolates were identified as $B$. cereus group strains using BLAST alignment. Then, the panC gene sequence confirmed that the 84 strains of the $B$. cereus group belonged to panC groups II, III, and IV. Of these, 7 isolates were assigned to group II (consisting of $B$. thuringiensis II and B. cereus s. s. II), 38 isolates to group III (a mixture group of $B$. thuringiensis III, $B$. cereus s. s. III, or B. anthracis), and 39 isolates to group IV (a mesophilic group of $B$. thuringiensis IV or B. cereus s. s. IV). We confirmed that the 84 B. cereus group isolates could be assigned to B. cereus s. s., B. thuringiensis, or B. anthracis.

Biochemical tests were performed to further identify the isolates (results are shown in Supplemental Figure S1; https://doi.org/10.3168/jds.2019-16392). A clear hemolysis circle around the colonies on blood agar medium (Supplemental Figure S1) indicated that the isolated strains had $\beta$-hemolytic activity. Furthermore, transparent hydrolyzed circles could be observed around colonies on casein medium, indicating that isolates could hydrolyze casein. Bacillus cereus s. s. possesses $\beta$-hemolytic and casein hydrolytic activities, whereas B. anthracis does not share these properties. Therefore, B. anthracis was deemed absent from the 84 isolates of the $B$. cereus group. Colonies on the manganese sulfate NA medium were then stained and observed under the microscope. Results showed that all isolates produced spores and none produced toxin crystals (Supplemental Figure S1). This indicates that these isolates did not belong to $B$. thuringiensis, which can produce parasporal crystals. In summary, the 84 isolates were thus identified as B. cereus s. s.

\section{MLST Analysis}

The sequences of 7 housekeeping genes of the $B$. cereus group were determined and referenced to the MLST database, with 3 novel alleles ( $p u r-242$, pyc-198, $i l v-276)$ detected. According to the ST of the isolates, which were determined based on the allelic coding of 7 housekeeping genes, these isolates clustered into 24 ST, including 14 novel ST, as shown in Table 2. The new ST were numbered from 1333 to 1345 and 1347, with corresponding ID from 2003 to 2015 and 2110. All of the new allele profiles and ST detected have been submitted to the $B$. cereus PubMLST database. The predominant ST was ST999 (19/84, 22.62\%), followed by ST1343 (13/84, 15.48\%), ST1335 (6/84, 7.14\%), and ST1345 (6/84, 7.14\%). The isolates of ST999 were mainly detected in the can filling and production areas, whereas ST1343 was most frequently associated with the big bag filling and production areas, as shown in Table 2 .

\section{Polymorphism Analysis}

Polymorphism analysis of the 7 housekeeping genes for the 84 B. cereus s. $s$. isolates is summarized in Table 3. The number of unique housekeeping genes alleles varied from $8(g m k)$ to $16(g l p F)$, and the number of polymorphic sites ranged from 21 (tpi) to 53 (pur). The nucleotide diversity ( $\pi$; the average number of nucleotide differences for each site between randomly selected sequences) varied from 0.0144 (tpi) to 0.0726 (ilvD). The $\mathrm{G}+\mathrm{C}$ content of the 7 housekeeping genes (from 38.2 to $44.7 \%$ ) was higher than the average $\mathrm{G}+\mathrm{C}$ content $(35 \%)$ of the whole-genome sequence of $B$. cereus group in the GenBank database (Liu et al., 2017). The value of Tajima's D ranged from -1.4997 to -0.7908 and the ratios of $\mathrm{Ka} / \mathrm{Ks}$ were much less than 1 for all loci, indicating that the sequence of housekeeping genes was conservative and stable. Thus, these genes were suitable for MLST analysis (Fei et al., 2015). 
Table 2. Multilocus sequence typing and traceability information of 84 Bacillus cereus sensu stricto isolates

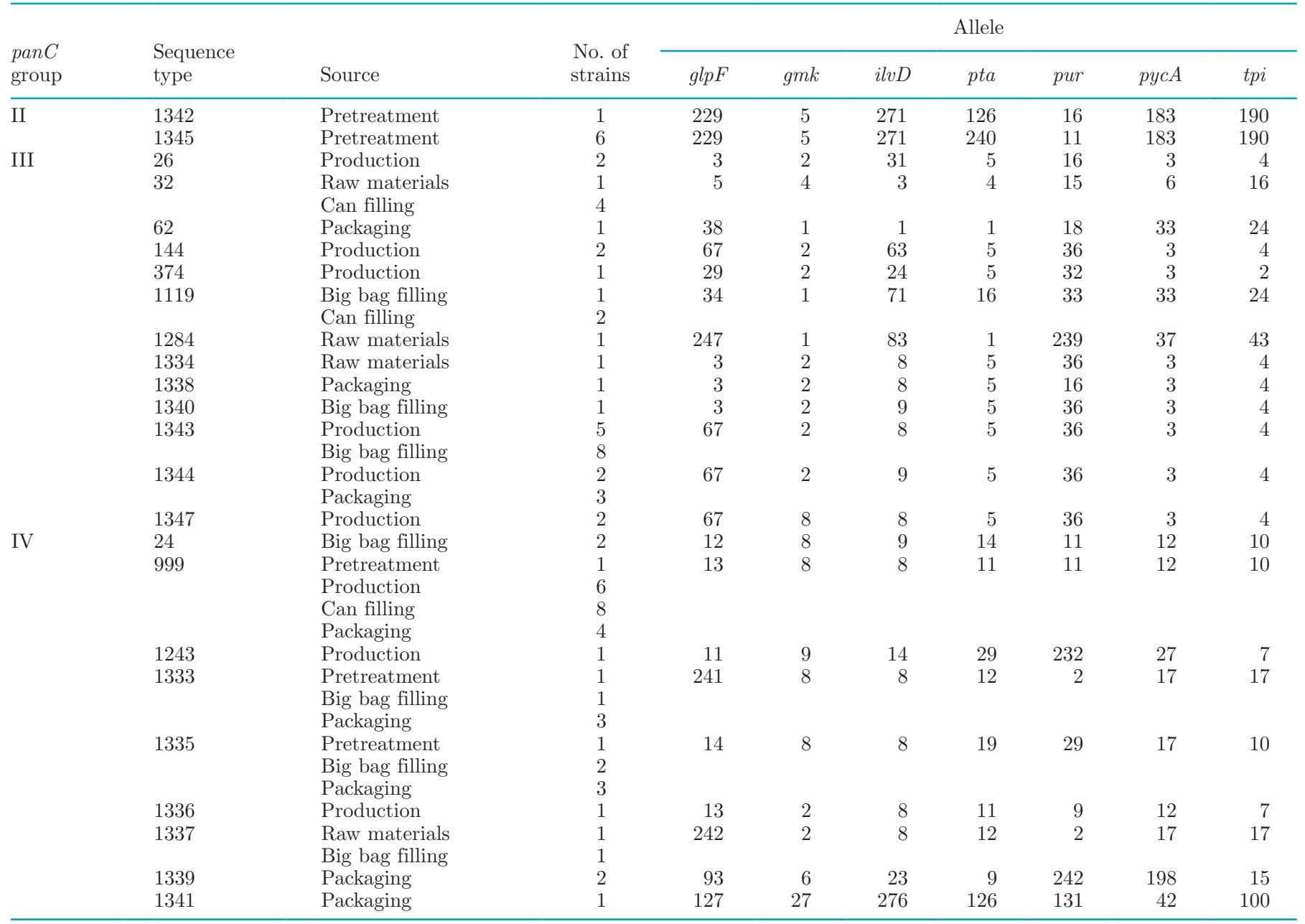

\section{Phylogenetic Analysis}

A maximum likelihood phylogenetic tree was constructed based on the concatenated sequences of the 7 housekeeping genes (total length 2,829 bp) for all 84 $B$. cereus $s$. $s$. isolates in this study and an additional 11 reference strains. The phylogenetic tree showed a clear phylogenetic relationship for the isolates (Figure $1)$. The $24 \mathrm{ST}$ clustered to the clade of $B$. cereus $s$. s. ATCC $14579^{\mathrm{T}}$, B. anthracis ATCC $14578^{\mathrm{T}}$, and $B$. thuringiensis ATCC $10792^{\mathrm{T}}$ and separately from the other 8 reference strains. As shown in Table 2, isolates with the same ST were classified into the same panC group, with some panC groups containing mul-

Table 3. Polymorphism of 7 housekeeping genes for 84 Bacillus cereus sensu stricto strains ${ }^{1}$

\begin{tabular}{|c|c|c|c|c|c|c|c|}
\hline Gene & $\begin{array}{l}\text { Size } \\
\text { (bp) }\end{array}$ & $\begin{array}{l}\text { No. of } \\
\text { alleles }\end{array}$ & $\begin{array}{l}\text { No. of polymorphic } \\
\text { sites }(\%)\end{array}$ & $\pi$ & $\begin{array}{c}\mathrm{G}+\mathrm{C} \\
\text { content }(\%)\end{array}$ & Tajima's D & $\mathrm{Ka} / \mathrm{Ks}$ \\
\hline$g l p F$ & 372 & 16 & 33 & 0.0177 & 38.3 & -1.4997 & 0.0759 \\
\hline pta & 414 & 13 & 27 & 0.0168 & 42.3 & -1.2807 & 0.0243 \\
\hline pur & 348 & 15 & 53 & 0.0502 & 38.6 & -1.0732 & 0.0086 \\
\hline pycA & 363 & 10 & 44 & 0.0694 & 41.1 & -1.3292 & 0.0234 \\
\hline
\end{tabular}

${ }^{1} \pi=$ nucleotide diversity (per site); Tajima's D = deviation from the standard neutral model of evolution; Ka $=$ the number of nonsynonymous substitutions per nonsynonymous site; $\mathrm{Ks}=$ the number of synonymous substitutions per synonymous site. 
tiple ST. The $24 \mathrm{ST}$ were divided into 3 panC gene groups. Group III was the largest group and included 13 ST (ST1119, ST62, ST1284, ST374, ST26, ST32, ST144, ST1338, ST1340, ST1334, ST1347, ST1343, and ST1344). Group II contained 2 ST: ST1342 and ST1345. Group IV included 9 ST (ST1341, ST1336, ST1337, ST1339, ST1243, ST1333, ST1335, ST24, and ST999).

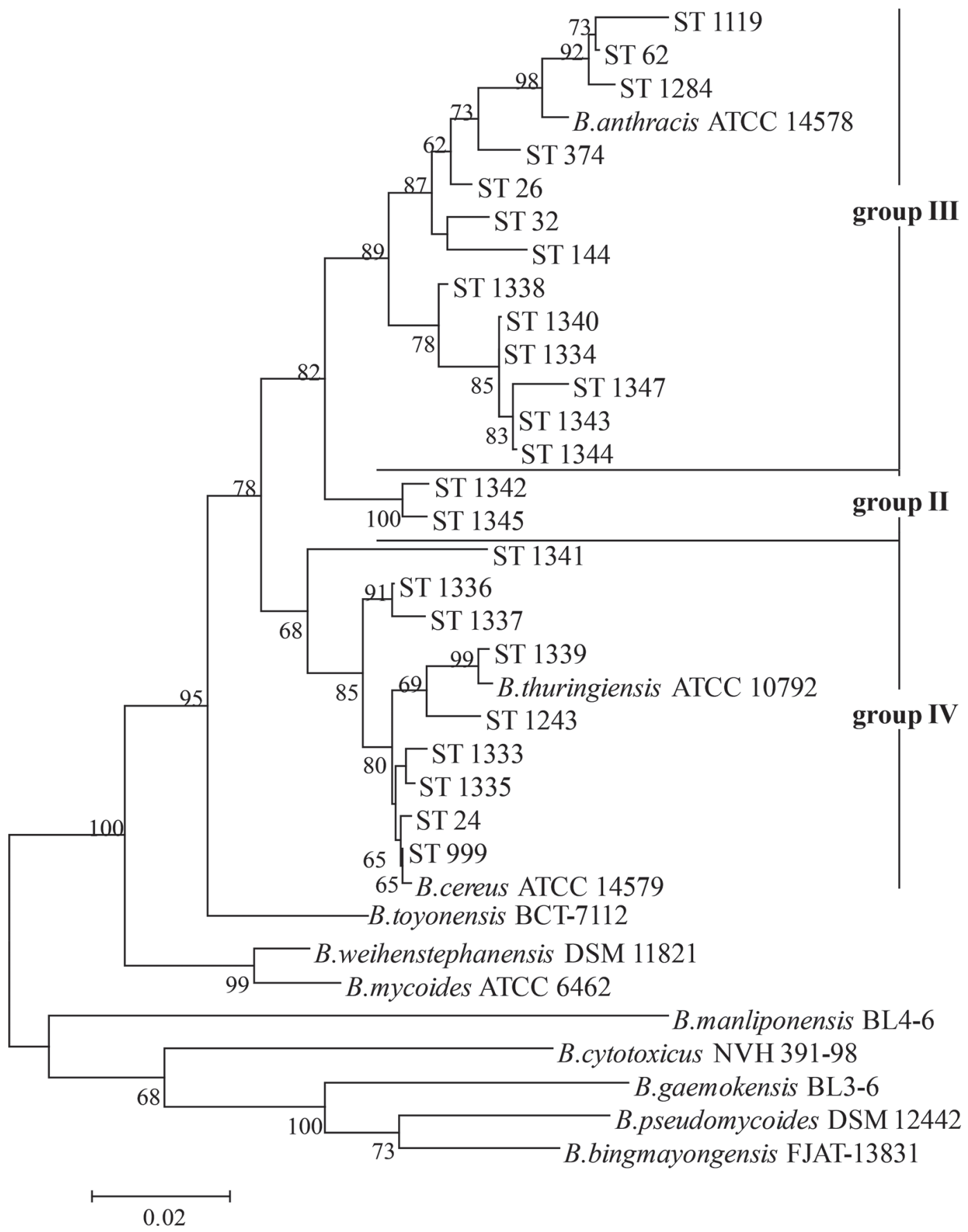

Figure 1. Maximum likelihood phylogenetic tree of the concatenated sequences (2,829 bp) of 7 housekeeping genes of the 84 Bacillus cereus sensu stricto isolates collected from raw materials and processing environments of the powdered infant formula (PIF) facility, and the Bacillus cereus group type strains $\left(B\right.$. cereus ATCC $14579^{\mathrm{T}}$, B. anthracis ATCC $14578^{\mathrm{T}}$, B. thuringiensis ATCC $10792^{\mathrm{T}}$, B. mycoides ATCC $6462^{\mathrm{T}}$, B. pseudomycoides DSM $12442^{\mathrm{T}}$, B. weihenstephanensis DSM $11821^{\mathrm{T}}$, B. cytotoxicus NVH 391-98 ${ }^{\mathrm{T}}$, B. toyonensis BCT-7112 ${ }^{\mathrm{T}}$, B. gaemokensis BL3- $6^{\mathrm{T}}$, B. manliponensis BL $4-6^{\mathrm{T}}$ and B. bingmayongensis FJAT-13831 ${ }^{\mathrm{T}}$ ). Bootstrap values were calculated based on 1,000 replicates. ST $=$ sequence type. 


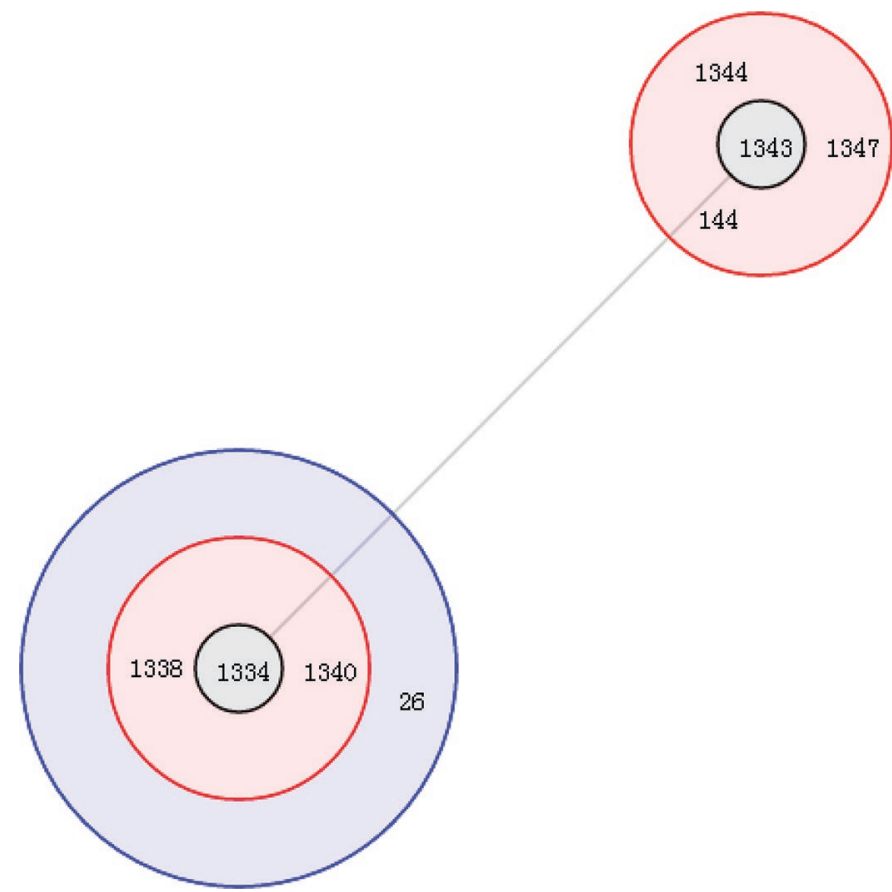

Figure 2. The clonal complexes (CC1343) classified by eBURST (http://pubmlst.org/bcereus/). Sequence type (ST)1343 was the founding ST of this CC, with the subgroup that contained ST1334 being closely related to this main group.

\section{Population Genetics Analysis}

Single and double locus variants were calculated using the eBURST online tool to investigate the relationship of the $24 \mathrm{ST}$ identified. These $24 \mathrm{ST}$ were divided into 4 clusters and 5 singletons. Cluster 1 included 6 ST (ST24, ST999, ST1333, ST1335, ST1336, and ST1337). Cluster 2 comprised 9 ST (ST26, ST144, ST374, ST1334, ST1338, ST1340, ST1343, ST1344, and ST1347). Two ST each belonged to cluster 3 (ST62, ST1119) and cluster 4 (ST1342 and ST1345). In addition, 5 ST (ST1284, ST32, ST1341, ST1339, and ST1243) were singletons. Among the 4 clusters, cluster 2 was a CC, with ST1343 being the central ST based on having the highest number of SLV (4 SLV and 2 DLV in total). As shown in Figure 2, ST1343 was the common ancestor ST of the CC, indicating that the other ST diverged from this ST. In addition, ST1334 included 3 SLV and 4 DLV and was assigned as the founder of a subgroup connected with the main CC group.

\section{Tracing Analysis}

With the exception of 4 isolates $(0.05 \%)$ from the raw materials, all others were isolated from the processing environment (Table 2). Among these 80 isolates, 10 were collected from the pretreatment area, 22 from the production area, 16 from big bag filling area, 14 from the can filling area, and the remaining 18 isolates from the packaging area. The correlations between sample sources and different $\mathrm{ST}$ of $B$. cereus s. s. are shown in Figure 3. Sequence type 32 was associated with the can filling area; ST1336, ST1343, ST1344, and ST1347 were associated with the production area; and ST1333, ST1334, ST1335, and ST1337 were associated with the pretreatment, packaging, big bag filling area, and raw material areas.

\section{Heat Resistance of Different ST of $B$. cereus sensu stricto}

In some cases, B. cereus s. s. can produce spores with high heat resistance. Results in Table 4 show that the $\mathrm{D}_{\mathrm{T}}$ values of different $\mathrm{ST}$ varied under the same temperature conditions, and the $\mathrm{D}_{\mathrm{T}}$ values of the same $\mathrm{ST}$ decreased with increasing temperature. Variation was also observed when comparing $\mathrm{ST}$ in individual panC gene groups. On average, the spores of group III were more heat resistant than those of the other 2 groups $(P<0.05)$. Among the $24 \mathrm{ST}$, the 9 with the greatest heat resistance phenotype for all 3 temperatures tested (ST62, ST1338, ST144, ST1284, ST26, ST1343,

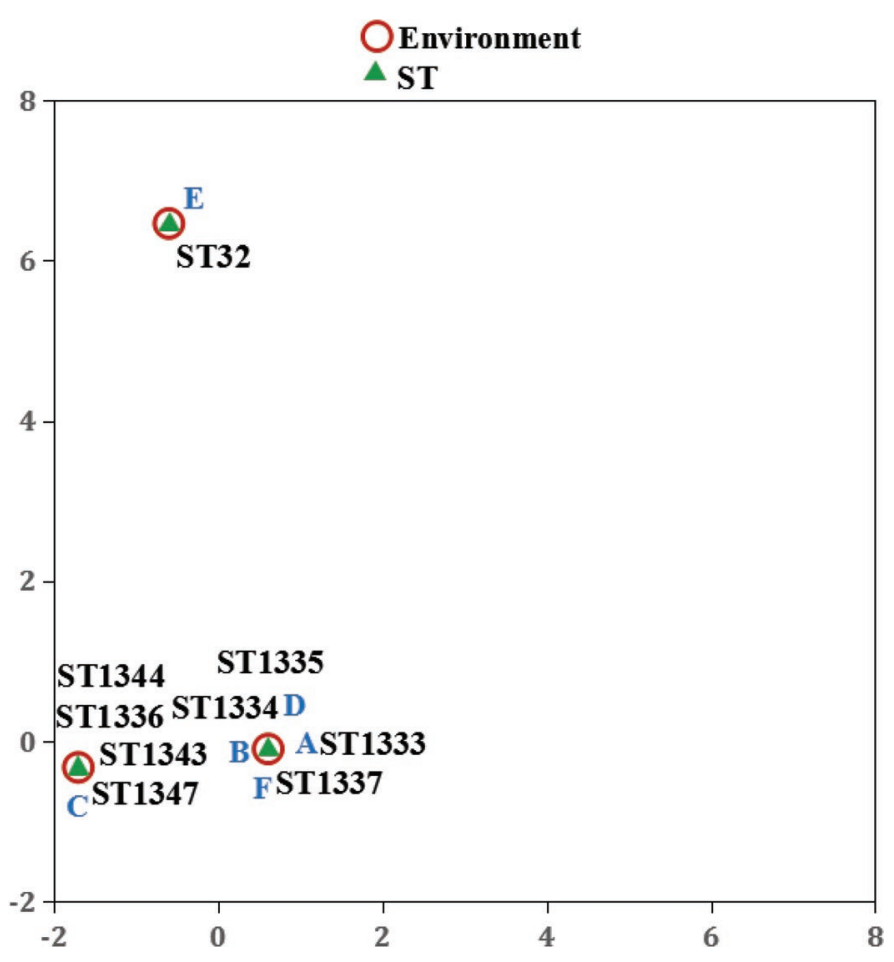

Figure 3. Correlation analysis between sequence type (ST) and powdered infant formula production environments: (A) raw materials area, (B) pretreatment area, (C) production area, (D) big bag filling area, $(\mathrm{E})$ can filling area, and $(\mathrm{F})$ packaging area. 


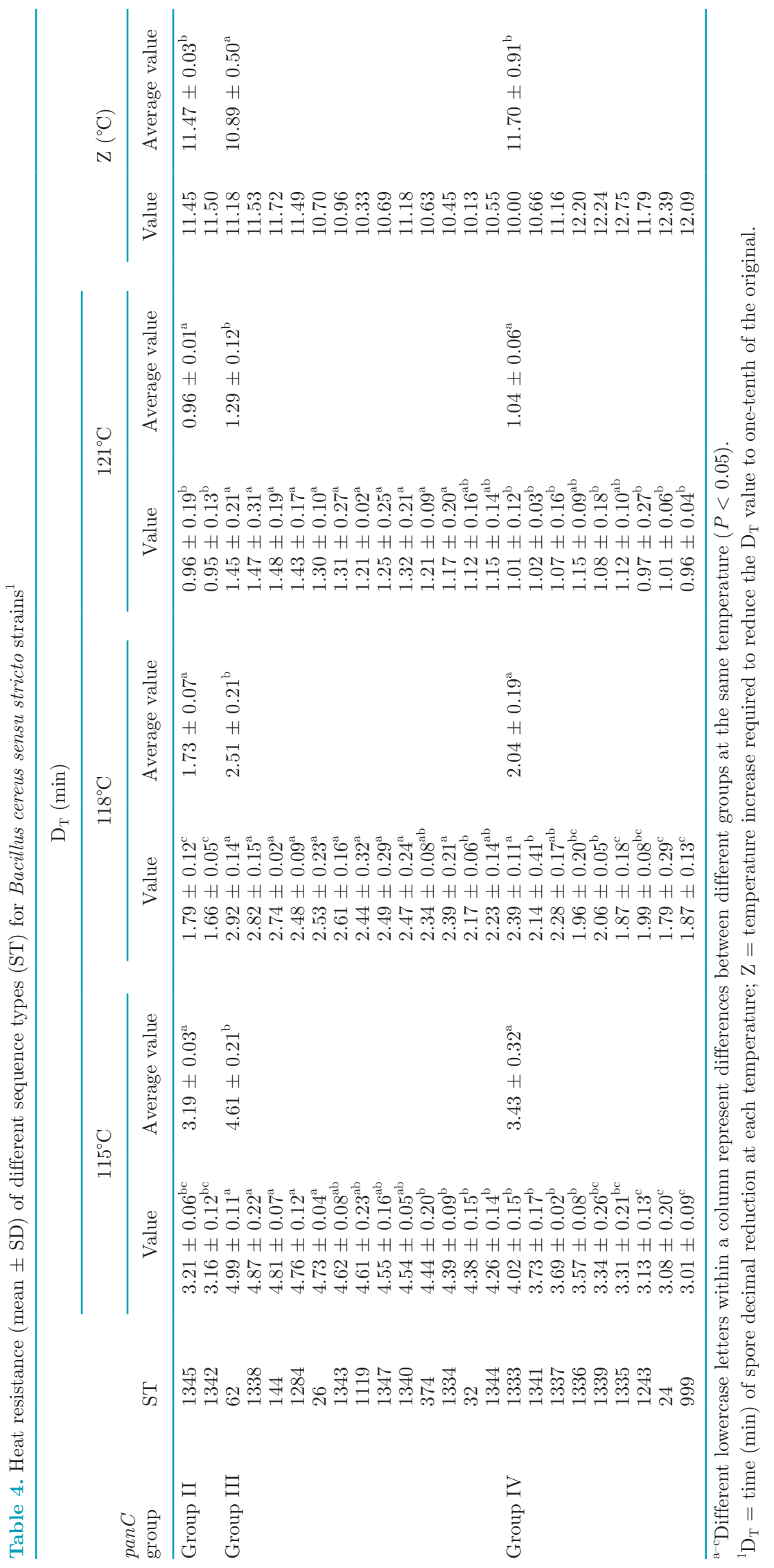


ST1119, ST1347, and ST1340) belonged to group III. In contrast, $\mathrm{D}_{\mathrm{T}}$ values of ST999, ST24, and ST1243 (attributed to group IV) were lowest, followed by ST1342 and ST1345 (members of group II). The heat resistance of group IV, although higher, on average, than that of group II, was not significantly different.

The spores from different phylogenetic groups had varying $\mathrm{Z}$ values. With the exception of ST1333, ST1341, and ST1337, spores of ST in group IV had higher Z values than those in the other 2 groups; ST1335, ST24, and ST1339 from group IV had the highest Z values among ST from all groups in our study. Although Z values between group IV and group II were not significantly different from each other, they were significantly higher than that of group III $(P<0.05)$.

\section{DISCUSSION}

The $B$. cereus group is an important foodborne pathogen that can be found in food products and the environment. The B. cereus group contains 11 species with high similarity in physiological and biochemical characteristics. Therefore, it is difficult to distinguish different species by a single phenotypic method or simple molecular method. Pink or white ring-like precipitation formed around colonies on MYP agar is used as a presumptive positive phenotype for typical strains of $B$. cereus group; however, this phenotype may be difficult to detect under a yellow background generated by other species present that utilize mannitol. Thus, suspected colonies on MYP agar should be confirmed and identified by molecular methods to avoid inaccurate detection results (Oliwa-Stasiak et al., 2011; Chon et al., 2015). Analysis of the 16S rRNA gene is a commonly used molecular tool for bacterial species identification, with BLAST comparison of the sequences demonstrating a close match to known $B$. cereus group sequences in the NCBI database confirming $B$. cereus group species. If the homology of sequences is greater than $99 \%$, the strain is highly likely to be the same species as the known sequence (McHugh et al., 2017). However, the genetic characteristics of various species in the $B$. cereus group are highly similar. For example, the $16 \mathrm{~S}$ rRNA sequences of $B$. cereus s. s., $B$. thuringiensis, and $B$. anthracis may differ by only a few bases, with an associated homology as high as 99\% (Liu et al., 2015). Therefore, $16 \mathrm{~S}$ rRNA gene analysis, while useful for confirmation at the $B$. cereus group level, may not distinguish at the species level.

The panC gene is one of the housekeeping genes for the $B$. cereus group, and is used in a classical taxonomy method for B. cereus group division (Ziane et al., 2014; Heini et al., 2018; Porcellato et al., 2019) to divide isolates into 7 groups (I to VII) according to different physiological and biochemical characteristics (Guinebretière et al., 2008). In this study, the 84 isolates were divided into 3 groups by pan $C$ gene analysis, including group II, III, and IV. These 3 groups consisted of $B$. cereus s. s., B. thuringiensis, and B. anthracis, allowing exclusion of the other 8 species of $B$. cereus group as potentially among the isolate cohort. We further distinguished these 3 species by the protease, hemolysis, and parasporal crystal tests. The results showed that all 84 stains were $B$. cereus s. s. In this study, molecular identification methods combined with physiological and biochemical tests eliminated the need for additional biochemical identification and reduced the time required for definitive speciation of isolates.

By using MLST, the 84 strains of $B$. cereus s. s. were divided into $24 \mathrm{ST}, 14$ of which were novel ST. This indicated that the B. cereus $s$. s. isolated from the raw materials and processing environments of PIF showed high genetic diversity. So far, the B. cereus MLST typing database contains more than 2,300 strains of $B$. cereus group, in 1,487 ST. In our study, 14 new ST contained 47 strains, accounting for $56 \%$ of the isolated strains, indicating that additional genotyping data should be collected for the MLST database.

Among the 24 ST in this study, ST999 was the most frequent type, shared by 19 isolates collected from the pretreatment, production, can filling, and packaging areas. This indicated that ST999 existed in most processing areas in this PIF facility. Based on eBURST analysis, ST999 and ST1336 belonged to the same cluster, which suggests that ST999 is closely related to ST1336 in phenotypic characteristics. Other ST have been reported in some studies, including those associated with carriage of the emetic toxin virulence gene ces. This toxin, a small and highly stable cyclic peptide, may be produced by B. cereus s. s. strains harboring the ces gene (Kumari and Sarkar, 2016). They are difficult to inactivate by standard food processing approaches or human gastric digestion once cereulide has formed in contaminated foods or associated ingredients (Ehling-Schulz et al., 2004; Han et al., 2015). Sequence type 26 and ST144, which have been associated with ces carriage, have been increasingly isolated from infant foods in recent years (Zhang et al., 2016; Yang et al., 2017). In this study, these 2 ST were part of clonal complex ST1343 and isolated from the production area and big bag filling area, belonging to pan $C$ gene group III. Sequence type 26 and ST144 were also isolated from infant formula, with previous work demonstrating that ST26 is the most common ancestor clone of B. cereus in infant formula from China (Yang et al., 2017).

In this study, eBURST analysis separated the 84 isolates in this study into 4 clusters and 5 singletons, with one of these clusters comprising a CC of which ST1343 
was inferred as the founder ST (Figure 2). This CC (CC1343) was the main group of B. cereus s. s. associated with the PIF processing environment and suggests these strains have a closer phylogenetic relationship. Clonal complex 1343 contained 9 ST and was one of the largest clusters represented in the MLST database. In addition, CC1434 (with ST1434 as core ST) may be of particular public health significance because it harbored isolates of the cereulide-associated subgroups ST26 and ST144. The sanitary conditions of processing environments must be strictly controlled in accordance with GMP (good manufacturing practices) and HACCP (hazard analysis critical control point) regulations, particularly the spray drying, fluidized bed, and packing processes, which are vulnerable to contamination. Secondary contamination of raw materials in processing environments should also be prevented. In addition, it is necessary to strengthen the supervision of infant formula production to prevent cross-contamination in different processing areas to ensure the quality and safety of products.

The phylogenetic tree based on the 7 housekeeping genes divided the 24 ST into 2 phylogenetic branches with high bootstrap values (Figure 1). These 2 main branches were further divided into smaller phylogenetic groups. Of the species included in this phylogenetic analysis, B. cereus s. s., B. anthracis, and B. thuringiensis showed a closer phylogenetic relationship; ST1340, ST1334, ST1347, ST1343, and ST1344 clustered relatively closely. These were mainly isolated from the production, big bag filling, and packaging areas, and all represent novel ST. Therefore, we speculate that there may be a risk of cross-contamination during the processing of infant formula in these 3 processing areas. Isolates from each of the panC groups identified among isolates in this study clustered separately into individual clades.

Tracing analysis can identify the possible contamination pathways of B. cereus s. s. during PIF processing and help direct efforts to reduce the contamination burden. The $84 \mathrm{~B}$. cereus s. s. isolates were unevenly distributed through the raw materials and PIF processing environments. Most of the B. cereus s. s. isolates $(80 / 84,95.24 \%)$ were from processing environments. Of these, most $(27.5 \%, 22 / 80)$ were obtained from the production area and the packing area (18/80, 22.5\%). This may indicate that the bacteria colonized niches in these areas such as the surface of the equipment, and human activities may be more frequent in these processing areas (Jacobs et al., 2011). Some studies have indicated that microorganisms can be associated with the shoes of employees, external roofs above spray driers, powder-packaging rooms, air treatment areas, and areas with high foot traffic in the processing environ- ments of PIF facilities, leading to cross-contamination into spray-drying facilities and the entry of pathogens into products (Craven et al., 2010; Sonbol et al., 2013). Previous tracing analysis of a UHT milk processing facility showed that most $B$. cereus isolates were associated with ingredient tanks, pasteurization tanks, and silo tanks, whereas few isolates were detected in final products (Lin et al., 2017). This suggested that B. cereus was inactivated during processing, with a smaller number of heat-resistant strains persisting.

The panC gene grouping scheme can be associated with the growth temperature range and heat resistance of $B$. cereus group isolates. In our study, the spores with the highest $\mathrm{D}_{\mathrm{T}}$ value belonged to group III, and the average $\mathrm{D}_{\mathrm{T}}$ value of this group was the highest of the 3 groups. This suggests that the heat resistance of group III was stronger than that of groups II and $\mathrm{IV}$, which is in agreement with the results obtained by Luu-Thi et al. (2014). We also reviewed $\mathrm{Z}$ value ranges of $B$. cereus spores from previous studies, which ranged from 8.3 to $12.8^{\circ} \mathrm{C}$; all of the $\mathrm{Z}$ values of spores from the $24 \mathrm{ST}$ in this study were within this range. Comparing the average $\mathrm{Z}$ value of 3 groups, group IV showed the highest value and group III the lowest, again mirroring results reported by Luu-Thi et al. (2014). Fiedoruk et al. (2017) found that the ribosomal proteins and heat resistance of $B$. cereus were relevant and well correlated with phylogenetic groups. Taken together, these results indicate that the heat resistance of spores from group III is higher and, as such, measures implemented for their control must account for this higher temperature resistance to reduce the contamination burden in dairy processing systems such as PIF manufacturing.

\section{CONCLUSIONS}

Samples collected from the raw materials and processing environments of PIF production facilities were analyzed by molecular biological and physiological and biochemical tests. Eighty-four B. cereus s. s. were detected. Only 4 of the $84 \mathrm{~B}$. cereus s. s. isolates were isolated from raw materials, indicating that the contamination of raw materials was lower than that in processing environments. The relationship between ST and processing environments can determine possible contamination pathways of B. cereus s. s. during PIF production. The spores from different panC gene groups varied in heat resistance, with the spores from group III showing higher resistance. PanC group III includes strains from all sampling areas with the exception of pretreatment. This demonstrates that temperature should be strictly controlled during all processing procedures to avoid the formation of spores. These results improve our understanding of $B$. cereus $s$. 
$s$. strains associated with dairy production systems and provide insights into strategies to control their presence in processing environments, including those associated with PIF production.

\section{ACKNOWLEDGMENTS}

This work was supported by the National Natural Science Foundation of China (31871828, Beijing) and the National High-Level Talents Special Support Program of China (810098, Beijing).

\section{REFERENCES}

Candelon, B., K. Guilloux, S. D. Ehrlich, and A. Sorokin. 2004. Two distinct types of rRNA operons in the Bacillus cereus group. Microbiology 150:601-611.

Castiaux, V., E. N'Guessan, I. Swiecicka, L. Delbrassinne, K. Dierick, and J. Mahillon. 2014. Diversity of pulsed-field gel electrophoresis patterns of cereulide-producing isolates of Bacillus cereus and Bacillus weihenstephanensis. FEMS Microbiol. Lett. 353:124-131.

Chon, J. W., J. H. Yim, H. S. Kim, D. H. Kim, H. Kim, D. H. Oh, S. K. Kim, and K. H. Seo. 2015. Quantitative prevalence and toxin gene profile of Bacillus cereus from ready-to-eat vegetables in South Korea. Foodborne Pathog. Dis. 12:795-799.

Craven, H. M., C. M. McAuley, L. L. Duffy, and N. Fegan. 2010. Distribution, prevalence and persistence of Cronobacter (Enterobacter sakazakii) in the nonprocessing and processing environments of five milk powder factories. J. Appl. Microbiol. 109:1044-1052.

Drewnowska, J. M., and I. Swiecicka. 2013. Eco-genetic structure of Bacillus cereus sensu lato populations from different environments in northeastern Poland. PLoS One 8:e80175.

Ehling-Schulz, M., M. Fricker, and S. Scherer. 2004. Identification of emetic toxin producing Bacillus cereus strains by a novel molecular assay. FEMS Microbiol. Lett. 232:189-195.

Esteban, M. D., J. P. Huertas, P. S. Fernandez, and A. Palop. 2013. Effect of the medium characteristics and the heating and cooling rates on the nonisothermal heat resistance of Bacillus sporothermodurans IC4 spores. Food Microbiol. 34:158-163.

Fei, P., C. Man, B. Lou, S. J. Forsythe, Y. Chai, R. Li, J. Niu, and Y. Jiang. 2015. Genotyping and source tracking of Cronobacter sakazakii and C. malonaticus isolates from powdered infant formula and an infant formula production factory in China. Appl. Environ. Microbiol. 81:5430-5439.

Fiedoruk, K., J. M. Drewnowska, T. Daniluk, K. Leszczynska, P. Iwaniuk, and I. Swiecicka. 2017. Ribosomal background of the Bacillus cereus group thermotypes. Sci. Rep. 7:46430.

Forsythe, S. J., B. Dickins, and K. A. Jolley. 2014. Cronobacter, the emergent bacterial pathogen Enterobacter sakazakii comes of age; MLST and whole genome sequence analysis. BMC Genomics 15:1121.

Giffel, M. C., and R. R. Beumer. 1999. Bacillus cereus: A review. J. Food Technol. Afr. 4:7-13.

Guinebretière, M. H., S. Auger, N. Galleron, M. Contzen, S. B. De, M. L. De Buyser, G. Lamberet, A. Fagerlund, P. E. Granum, and D. Lereclus. 2013. Bacillus cytotoxicus sp. nov. is a novel thermotolerant species of the Bacillus cereus group occasionally associated with food poisoning. Int. J. Syst. Evol. Microbiol. 63:31-40.

Guinebretière, M. H., F. Thompson, A. Sorokin, P. Normand, P. Dawyndt, M. Ehling-Schulz, B. Svensson, V. Sanchis, C. Nguyen, and M. Heyndrickx. 2008. Ecological diversification in the Bacillus cereus group. Environ. Microbiol. 10:851-865.

Hammerum, A. M., F. Hansen, N. M. Skov, M. Stegger, S. P. Andersen, A. Holm, L. Jakobsen, and S. U. Justesen. 2015. Investigation of a possible outbreak of carbapenem-resistant Acinetobacter baumannii in Odense, Denmark using PFGE, MLST and wholegenome-based SNPs. J. Antimicrob. Chemother. 70:1965-1968.
Han, H., T. Sullivan, and A. C. Wilson. 2015. Cytochrome c551 and the cytochrome c maturation pathway affect virulence gene expression in Bacillus cereus ATCC 14579. J. Bacteriol. 197:626-635.

Hayrapetyan, H., T. Abee, and M. N. Groot. 2016. Sporulation dynamics and spore heat resistance in wet and dry biofilms of Bacillus cereus. Food Control 60:493-499.

Heini, N., R. Stephan, M. Ehling-Schulz, and S. Johler. 2018. Characterization of Bacillus cereus group isolates from powdered food products. Int. J. Food Microbiol. 283:59-64.

Jacobs, C., P. Braun, and P. Hammer. 2011. Reservoir and routes of transmission of Cronobacter spp. in a milk powder-producing plant. J. Dairy Sci. 94:3801-3810.

Jung, M. Y., J. S. Kim, W. K. Paek, J. Lim, H. Lee, P. I. Kim, J. Y Ma, W. Kim, and Y. H. Chang. 2011. Bacillus manliponensis sp. nov., a new member of the Bacillus cereus group isolated from foreshore tidal flat sediment. J. Microbiol. 49:1027-1032.

Kent, R. M., G. F. Fitzgerald, C. Hill, C. Stanton, and R. P. Ross. 2015. Novel approaches to improve the intrinsic microbiological safety of powdered infant milk formula. Nutrients 7:1217-1244.

Kumari, S., and P. K. Sarkar. 2016. Bacillus cereus hazard and control in industrial dairy processing environment. Food Control 69:20-29.

Lechner, S., R. Mayr, K. P. Francis, B. M. Prüss, T. Kaplan, E. Wiessnergunkel, G. S. Stewart, and S. Scherer. 1998. Bacillus weihenstephanensis sp. nov. is a new psychrotolerant species of the Bacillus cereus group. Int. J. Syst. Bacteriol. 48:1373-1382.

Lin, Y., F. Ren, L. Zhao, and H. Guo. 2017. Genotypes and the persistence survival phenotypes of Bacillus cereus isolated from UHT milk processing lines. Food Control 82:48-56.

Liu, Y., W. Ge, J. Zhang, X. Li, X. Wu, T. Li, X. Zhang, and X. Wang. 2018. Detection of Bacillus cereus sensu lato from environments associated with goat milk powdered infant formula production facilities. Int. Dairy J. 83:10-16.

Liu, Y., Q. Lai, J. Du, and Z. Shao. 2017. Genetic diversity and population structure of the Bacillus cereus group bacteria from diverse marine environments. Sci. Rep. 7:689.

Liu, Y., Q. Lai, M. Göker, J. P. Meierkolthoff, M. Wang, Y. Sun, L. Wang, and Z. Shao. 2015. Genomic insights into the taxonomic status of the Bacillus cereus group. Sci. Rep. 5:14082.

Luu-Thi, H., D. B. Khadka, and C. W. Michiels. 2014. Thermal inactivation parameters of spores from different phylogenetic groups of Bacillus cereus. Int. J. Food Microbiol. 189:183-188.

Mafart, P., O. Couvert, S. Gaillard, and I. Leguérinel. 2002. On calculating sterility in thermal preservation methods: Application of the Weibull frequency distribution model. Int. J. Food Microbiol. $72: 107-113$.

Maiden, M. C., M. J. J. Van Rensburg, J. E. Bray, S. G. Earle, S. A. Ford, K. A. Jolley, and N. D. McCarthy. 2013. MLST revisited: The gene-by-gene approach to bacterial genomics. Nat. Rev. Microbiol. 11:728

McHugh, A. J., C. Feehily, C. Hill, and P. D. Cotter. 2017. Detection and enumeration of spore-forming bacteria in powdered dairy products. Front. Microbiol. 8:109.

Mckillip, J. L., P. Nimmer, and M. R. Beer. 2014. Bacillus cereus: A bacterial species of environmental and clinical significance. J. Liberal Arts. Sci. 18:21-32.

Oliwa-Stasiak, K., O. Kolaj-Robin, and C. C. Adley. 2011. Development of real-time PCR assays for detection and quantification of Bacillus cereus group species: differentiation of $B$. weihenstephanensis and rhizoid B. pseudomycoides isolates from milk. Appl. Environ. Microbiol. 77:80-88.

Otlewska, A., E. Oltuszak-Walczak, and P. Walczak. 2013. Differentiation of strains from the Bacillus cereus group by RFLP-PFGE genomic fingerprinting. Electrophoresis 34:3023-3028.

Patiño-Navarrete, R., and V. Sanchis. 2017. Evolutionary processes and environmental factors underlying the genetic diversity and lifestyles of Bacillus cereus group bacteria. Res. Microbiol. 168:309-318.

Pérez-Losada, M., P. Cabezas, E. Castro-Nallar, and K. A. Crandall. 2013. Pathogen typing in the genomics era: MLST and the future of molecular epidemiology. Infect. Genet. Evol. 16:38-53. 
Porcellato, D., M. Aspholm, S. B. Skeie, and H. Mellegård. 2019. Application of a novel amplicon-based sequencing approach reveals the diversity of the Bacillus cereus group in stored raw and pasteurized milk. Food Microbiol. 81:32-39.

Priest, F. G., M. Barker, L. W. Baillie, E. C. Holmes, and M. C. Maiden. 2004. Population structure and evolution of the Bacillus cereus group. J. Bacteriol. 186:7959-7970.

Santos, A. S., J. J. Tilburg, A. Botelho, M. J. Barahona, M. S. Núncio, M. H. Nabuurs-Franssen, and C. H. Klaassen. 2012. Genotypic diversity of clinical Coxiella burnetii isolates from Portugal based on MST and MLVA typing. Int. J. Med. Microbiol. 302:253-256.

Scheldeman, P., L. Herman, S. Foster, and M. Heyndrickx. 2006. Bacillus sporothermodurans and other highly heat-resistant spore formers in milk. J. Appl. Microbiol. 101:542-555.

Sonbol, H., S. Joseph, C. M. McAuley, H. M. Craven, and S. J. Forsythe. 2013. Multilocus sequence typing of Cronobacter spp. from powdered infant formula and milk powder production factories. Int. Dairy J. 30:1-7.

Teh, K. H., S. Flint, J. Palmer, P. Andrewes, P. Bremer, and D. Lindsay. 2012. Proteolysis produced within biofilms of bacterial isolates from raw milk tankers. Int. J. Food Microbiol. 157:28-34.
Wu, S., Y. Jiang, B. Lou, J. Feng, Y. Zhou, L. Guo, S. J. Forsythe, and C. Man. 2018. Microbial community structure and distribution in the air of a powdered infant formula factory based on cultivation and high-throughput sequence methods. J. Dairy Sci. 101:69156926.

Yang, Y., X. Yu, L. Zhan, J. Chen, Y. Zhang, J. Zhang, H. Chen, Z. Zhang, Y. Zhang, Y. Lu, and L. Mei. 2017. Multilocus sequence type profiles of Bacillus cereus isolates from infant formula in China. Food Microbiol. 62:46-50.

Zahner, V., A. C. T. D. C. Silva, G. P. D. Moraes, D. McIntosh, and I. D. Filippis. 2013. Extended genetic analysis of Brazilian isolates of Bacillus cereus and Bacillus thuringiensis. Mem. Inst. Oswaldo Cruz 108:65-72.

Zhang, Z., L. Feng, H. Xu, C. Liu, N. P. Shah, and H. Wei. 2016. Detection of viable enterotoxin-producing Bacillus cereus and analysis of toxigenicity from ready-to-eat foods and infant formula milk powder by multiplex PCR. J. Dairy Sci. 99:1047-1055.

Ziane, M., N. Desriac, P. Le Chevalier, O. Couvert, B. Moussa-Boudjemaa, and I. Leguerinel. 2014. Identification, heat resistance and growth potential of mesophilic spore-forming bacteria isolated from Algerian retail packaged couscous. Food Control 45:16-21. 